\title{
Revision Hip Arthroplasty
}

\section{Infection is the Most Common Cause of Failure}

\author{
S. Mehdi Jafari MD, Catelyn Coyle BA, \\ S. M. Javad Mortazavi MD, Peter F. Sharkey MD, \\ Javad Parvizi MD, FRCS
}

Published online: 3 March 2010

(C) The Association of Bone and Joint Surgeons $\mathbb{R} 2010$

\begin{abstract}
Background Revision total hip arthroplasty (THA), although relieving pain and restoring function, fails in some patients. In contrast to failures in primary THA, the frequency of the causes of failure in revision THA has been less well established.

Questions/purposes We therefore determined the rate of each failure mode and the survivorship of revision THAs. Methods We retrospectively reviewed the charts of 1366 revision THAs performed between 2000 and 2007. There were $609(44.5 \%)$ men and $757(55.5 \%)$ women with a mean age of 66 years. The indications for the revision surgery were mainly aseptic loosening (51\%), instability $(15 \%)$, wear (14\%), and infection (8\%). The minimum followup was 1 day (mean, 5.5 years; range, 1 day to 9 years).

Results Two hundred fifty-six of the revisions (18.7\%) failed with an average time to failure of 16.6 months (range, 1 day to 7.5 years). Among 256 failed hips, infection was the most common cause of failure (30.2\%) followed by
\end{abstract}

Each author certifies that he or she has no commercial associations (e.g, consultancies, stock ownership, equity interest, patent/licensing arrangements, etc.) that might pose a conflict of interest in connection with the submitted article.

Each author certifies that his or her institution approved the human protocol for this investigation, and that all investigations were conducted in conformity with ethical principles of research.

S. M. Jafari, C. Coyle, P. F. Sharkey, J. Parvizi ( $\square)$

Department of Orthopaedic Surgery, Rothman Institute at Thomas Jefferson University, 925 Chestnut Street, Philadelphia, PA 19107, USA

e-mail: parvj@aol.com

\section{S. M. J. Mortazavi}

Department of Orthopaedic Surgery, Imam University Hospital, Tehran University of Medical Sciences, End of keshavarz Blvd, Tehran 1419733141, Iran instability (25.1\%) and aseptic loosening (19.4\%). At 5 years, the survivorships of septic and aseptic groups were $67 \%$ and $84.8 \%$, respectively. Revision for infection or instability appears to have a considerably lower survivorship when compared to revision for aseptic causes.

Conclusions The lower survivorship of revision for infection or instability highlights the importance of implementing better preventative methods that can minimize the impact of these two major causes of failure.

Level of Evidence Level III, therapeutic study. See the Guidelines for Authors for a complete description of levels of evidence.

\section{Introduction}

Revision of a failed THA is a challenge to orthopaedic surgeons and frequently requires high levels of hospital resources [7]. In recent years, a steady increase in the number of revision THAs has been observed in the United States [6, 17]. As the number of primary THAs increases, so will the number of subsequent revision procedures. This number is expected to grow substantially over the next several decades $[6,12]$. Generally, the longevity of revision THAs is lower than that of primary THAs [15]. In long term ( $\geq 12$ years) survivorship between 60 to $81 \%$ for cementless revision THA cases is reported $[8,16,20]$. In a large-scale study by Lie et al. on 4762 revision THAs, the 10-year risk of failure for a revision operation with no prior infection was $25.6 \%$ [15]. If they had included the septic revisions, the failure rates would likely have been even higher. In another study by Springer et al. the survivorship following revision arthroplasty was $81 \%$ at 11 years for infected cases and $81 \%$ at 10 years for revisions done for aseptic loosening [20]. However, in contrast to failures in 
primary THA, the frequency of the causes of failure in revision THA has been less well established.

We asked the following questions: (1) What is the rate of each failure mechanism following revision THA? (2) How does the indication of index revision surgery affect the survivorship? (3) How frequently is the mode of failure of a revision the same as the indication for the index revision?

\section{Patients and Methods}

Subsequent to the approval of the Institutional Review Board, we gathered the data using our institutional database. We retrospectively reviewed the data from 1272 patients (1366 hips) who underwent revision THA between January 2000 and January 2007. We defined an operation as revision THA when any part of the hip prosthesis, including the acetabular component (shell or cage), acetabular liner, femoral stem, and/or femoral head, was exchanged. Twostage revision THA for infection was also included. Since the criteria for the diagnosis of infected hip replacement is evolving, the surgeons at our institution at any time use the contemporaneous criteria which are based on clinical presentation, imaging, serology, histopathology, bacteriology and recently sophisticated modalities such as PCR for questionable cases. For the current study periprosthetic infection was assumed to be the main cause of failure when the following criteria were present: abnormal serology, presence of sinus tract, evidence of purulence at surgery, and isolation of an organism from aspiration and/or intraoperative culture [5]. There were 587 men (46\%) and 685 women (54\%). Six hundred twenty-one (45\%) were left hips and 745 $(55 \%)$ were right hips. Four patients underwent bilateral revision THA in one session. The mean age of the patients was 66 years (range, 24-94 years) and their average body mass index (BMI) was $28.2 \mathrm{~kg} / \mathrm{m}^{2}$ (range, 16.1-53.8 kg/ $\mathrm{m}^{2}$ ). The diagnoses that resulted in index revision surgery included aseptic loosening in $694(51 \%)$, instability in 203 $(15 \%)$, wear in $190(14 \%)$, infection in $112(8 \%)$, periprosthetic fracture in 73 (5\%), and miscellaneous in 94 (7\%) hips (Fig. 1). Of the miscellaneous cases, there was osteolysis in 37 , component fracture in 24 (stem fracture in 19 and ceramic head fracture in five), limb length discrepancy in 28 , and squeaking in 5 hips. Of the 694 hips with aseptic loosening, $307(44 \%)$ had just loosening of the acetabular component, $249(36 \%)$ had loosening of the femoral component alone, and $138(20 \%)$ had loosening of both acetabular and femoral components. The minimum followup was 1 day (mean, 5.5 years; range, 1 day to 9 years). We tried to contact all the patients to find out whether they had reoperation or rerevision for any reason in another location. Of our cohort of 1272 patients, 65 (5\%) were lost to followup: 53 patients had no accessible contact information and 12 were not willing to

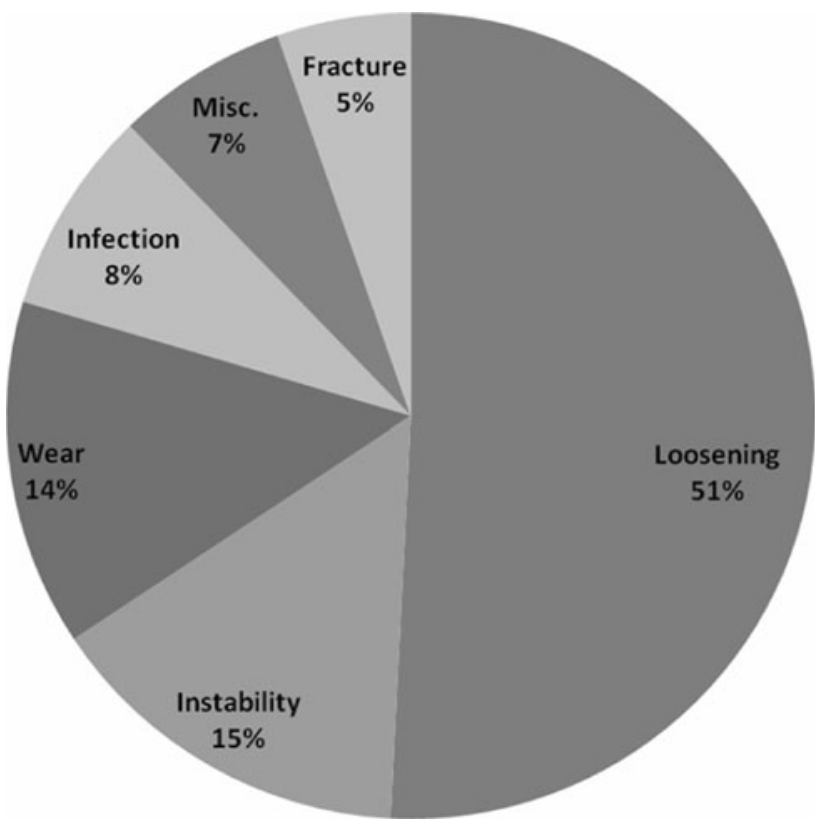

Fig. 1 This chart presents the rates of indications of index revision hip arthroplasty. Aseptic loosening is the major indication for revision THA.

discuss their status. This was a chart review study and no patients were seen in followup specifically for the study.

Operations were carried out in the supine position through a direct lateral approach. During the revision surgery for $135(10 \%)$ hips, trochanteric osteotomy and fixation were performed. Cementless fixation of both components was used in 1009 (74\%) revisions, cemented fixation of both components was used in $339(25 \%)$ revisions, and hybrid fixation (cemented fixation of one component and cementless fixation of the other component) was used in $18(1 \%)$ revisions. During the revision surgery of 21 hips in the instability group, failure of the locking mechanism of the acetabular component was encountered. For 348 (25\%) of 1366 revisions, bone graft was inserted in the femoral and/or acetabular side. In 69 hips, an acetabular cage was inserted. A 22-, 28-, 32-, or $36-\mathrm{mm}$ head was used in all cases. Fifteen patients received a $22-\mathrm{mm}$ head, 51 patients received a $28-\mathrm{mm}$ head, 667 patients received a $32-\mathrm{mm}$, and 633 patients received a 36$\mathrm{mm}$ head. Ceramic ball heads were used in 32 hips and cobalt-chrome ball heads were inserted in 1334 hips. In 83 cases, constrained liners were used. In 152 hips, a $10^{\circ}$ or $20^{\circ}$ lipped acetabular liner was inserted.

The regimen for thromboprophylaxis consisted of administration of warfarin on the day of surgery and continued for 6 weeks aiming for an international normalized ratio of 1.8 to 2.0. Patients were given intravenous firstgeneration cephalosporin or vancomycin for those with an allergy before the skin incision and for $24 \mathrm{~h}$ postoperatively. Patients were generally mobilized within 2 days 
after surgery under the supervision of a physiotherapist. On discharge, $556(40.7 \%)$ of patients were sent home and 805 $(58.9 \%)$ of them were sent to a rehabilitation facility. Five patients $(0.3 \%)$ died during the hospital stay. Partial weightbearing with crutches continued for 6 weeks. Afterward the crutches were discarded and full weightbearing was permitted.

The patients were routinely followed clinically and radiographically at 6 weeks, 6 months, 2 years, and then every 2 years postoperatively. AP and lateral radiographs are assessed by the senior attending physician for signs of failures (loosening, infection, wear, etc.). Patients are followed until the time of failure or censorship (death or loss to followup).

We considered the date of rerevision or reoperation as the end point and the period from the index revision procedure to the end point as time to failure. Furthermore, we divided the failures into early and late groups based on the time to failure after the surgery. The failures that occurred less or more than 2 years after surgery were considered as early and late failures, respectively. Modes of failure were determined by the surgeon and then we categorized them into six groups, including aseptic loosening, instability, wear, infection, periprosthetic fracture, and miscellaneous. Since only one hip failed due to osteolysis we categorized it in the miscellaneous group. If multiple modes of failure were evident, we considered the predominant mechanism as noted by the surgeon on the operation note. For understanding the mode of failure, preoperative clinical and radiographic findings along with intraoperative findings such as status of the components and also culture results were reviewed.

We calculated standard descriptive statistics, including average, range, frequency, and proportions for gender, age, BMI, laterality, indication of index revision surgery, failure mechanisms, and time to failure. We used Kaplan-Meier survivorship analysis to estimate the probability of survival of the revision surgery from the time of the index revision to the end point (failure) [9, 11]. First we separated the cohort into septic and aseptic groups based on the indication of index revision surgery; then, we compared the survivorship of these two groups by using the log rank test. Second we separated the cohort into three groups: septic, instability, and the others, and again compared their survivorship by using the log rank test. All survivorship data were reported with $95 \%$ confidence intervals (CIs). We used SPSS ${ }^{\circledR}$ for Windows ${ }^{\circledR} 15.0$ (SPSS Inc, Chicago, IL) for statistical analysis.

\section{Results}

With reoperation or rerevision as the end point, at a minimum followup of 1 day (mean, 5.5 years; range, 1 day to
9 years), 256 (18.7\%) of 1366 revision THAs failed including 73 reoperations and 183 rerevisions. Average time to failure after the index revision procedure was 16.6 months (range, 1 day to 7.5 years). Infection comprised approximately one-third of the failures (77 [30\%]) followed by instability (63 [25\%]), aseptic loosening (49 [19\%]), periprosthetic fracture (38 [15\%]), and others (29 [11\%]) (Fig. 2). During the period of followup, there were no failures as a consequence of wear. The failures were early (less than 2 years) in 197 hips and late (greater than 2 years) in 59 hips. Of 77 septic failures $71(92 \%)$ were early and six $(8 \%)$ were late. Of 63 failures resulting from instability, $51(81 \%)$ happened early and 12 (19\%) happened late. Of 49 loosenings $26(53 \%)$ occurred early and $23(47 \%)$ occurred late.

Overall survivorship at 5 years was $83.3 \%$ (95\% CI, 81.1-85.5) (Fig. 3). At 5 years, the survivorship of the septic group was $67 \%$ (95\% CI, 57.8-76.2) and that of the aseptic group was $84.8 \%$ (95\% CI, 82.6-87) (Fig. 4). Septic revisions had a lower survival probability $(\mathrm{p}<0.0001)$ compared with aseptic ones. At 5 years, the survivorship of the instability group was $75.9 \%$ (95\% CI, $69.8 \%-81.9 \%$ ), and that of the others group after separation of the instability group was $95.6(95 \% \mathrm{CI}, 94.6 \%$ 96.5\%) (Fig. 5). Revision THAs for infection and instability was associated with reduced survivorship $(p<0.0001)$ compared with the revisions for other reasons.

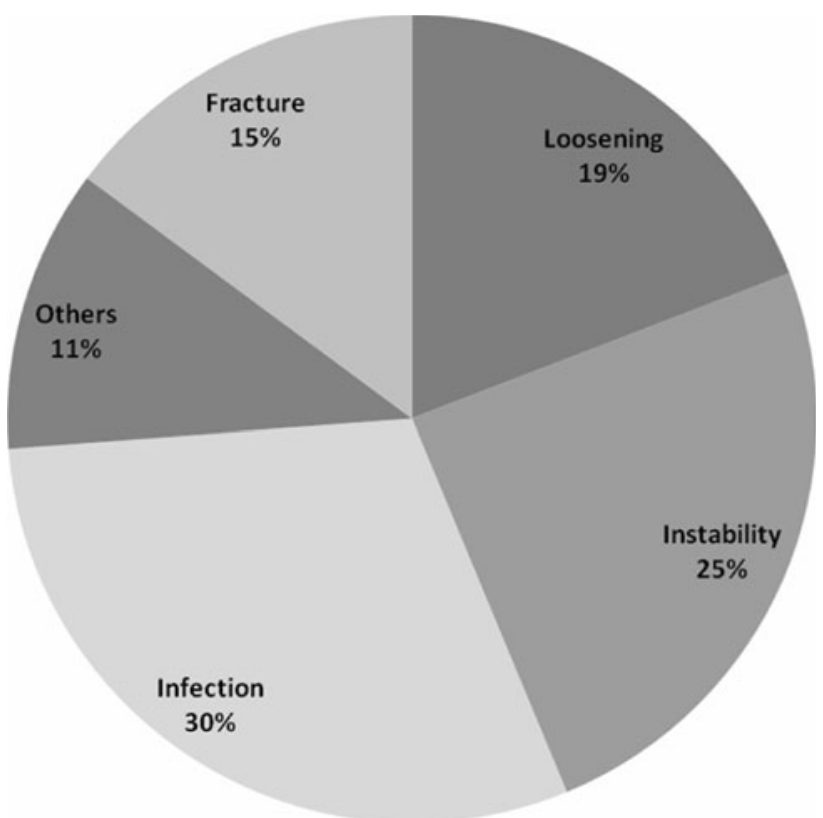

Fig. 2 This chart presents the rate of failure mechanisms following index revision hip arthroplasty. Infection and instability are the two most common causes of failure, accounting for $55 \%$ of failures combined. 


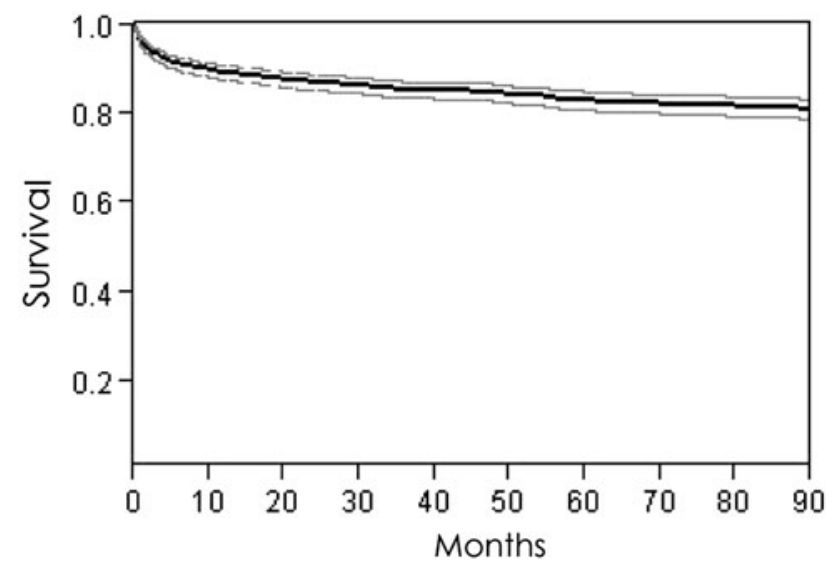

Fig. 3 According to the Kaplan-Meier analysis, overall survivorship at 5 years was $83.3 \%$ (95\% confidence interval, 81.1-85.5).

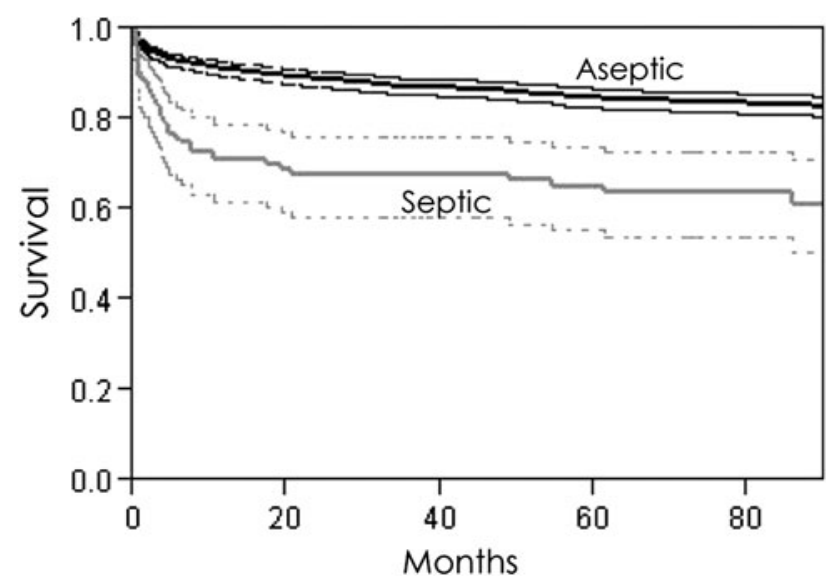

Fig. 4 The comparison of the survivorship between septic and aseptic groups is shown in this figure. According to the Kaplan-Meier analysis, at 5 years the survivorship of septic revisions (67\%) was less $(\mathrm{p}<0.0001)$ compared to the survival of the aseptic ones $(84.8 \%)$.

Starting from 1366 hip revision cases, of 652 revisions performed for aseptic loosening, 23 (4\%) failed because of aseptic loosening again, $74(11 \%)$ failed as a result of causes other than aseptic loosening, and 555 (85\%) succeeded (Fig. 6). Of 202 revisions performed for instability $29(14 \%)$ failed because of instability again, $22(11 \%)$ failed as a result of other than instability causes, and 151 (75\%) succeeded. Of 189 revisions performed for wear, none failed because of wear again, $21(11 \%)$ failed as a result of causes other than wear, and 168 (89\%) succeeded. Of 112 revisions for infection, 25 (22\%) failed because of infection again (infection persisted), 21 (19\%) failed as a result of other causes, and $66(59 \%)$ succeeded. Of 73 revisions for periprosthetic fracture, four $(5 \%)$ failed because of periprosthetic fracture again, $10(14 \%)$ failed as a result of the causes other than periprosthetic fracture, and $59(81 \%)$ succeeded.

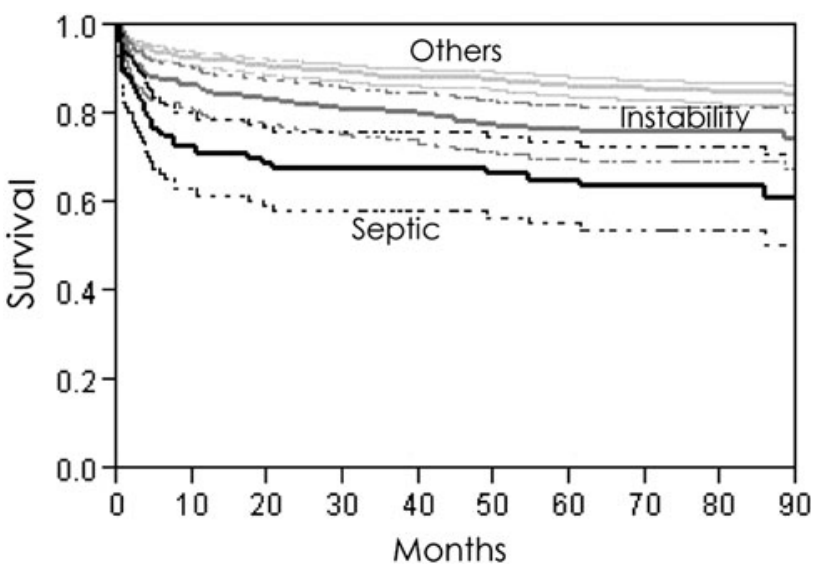

Fig. 5 This figure depicts the comparison of the survivorship of the three separated groups: septic, instability, and the others. According to the Kaplan-Meier analysis, the survivorship of the septic and instability groups at 5 years were $67 \%$ and $75.9 \%$, respectively, which was less $(\mathrm{p}<0.0001)$ than the survival rate of the revisions performed for other reasons $95.6 \%$.

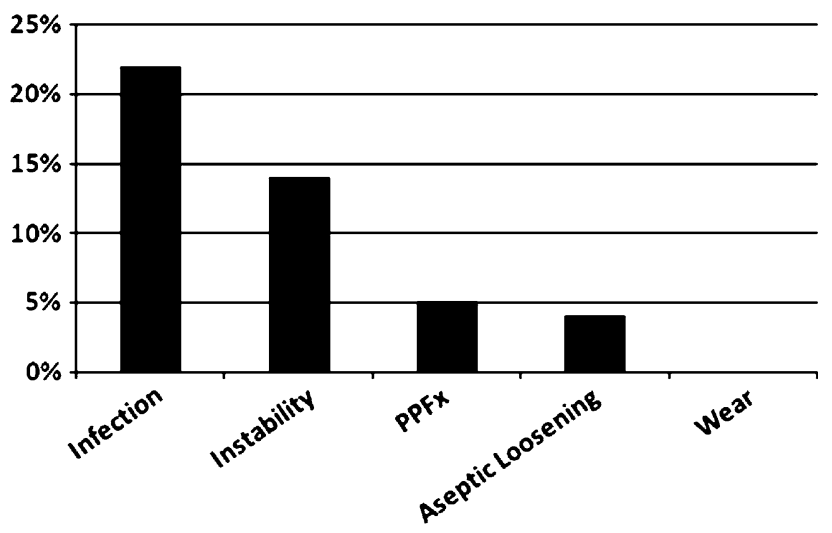

Fig. 6 This figure shows the frequency with which the failure modes of the revisions were the same as the indications for the index revisions.

\section{Discussion}

Revision THA is a complex, time-consuming, and technically challenging procedure with substantially different resource requirements than primary THA [7]. Revision procedures are more difficult and associated with greater liability for the surgeon $[3,7,19]$. Moreover, operative time is usually longer, bone loss is more frequent, and length of hospital stay and postoperative complication rates are higher $[2,4,7,13,14,17]$. While the causes and frequencies of failure of primary THA are well established, the frequencies of the cause of failure of revision THA are less well established. We determined: (1) the frequency of failure mechanisms after revision THA; (2) the survivorship of revision surgeries performed for infection or instability compared to those performed for other reasons; and (3) the persistence of failure mechanisms, especially if 
the index revision was performed for infection or instability.

The following are some limitations in our study that warrant mentioning. First, this study is conducted based on a retrospective review of a prospectively collected database. Thus variability in data collection and potential bias may have existed. The variability in data collection relates to missing data on details of intra-operative findings for some patients. Potential bias relates to difference in followup in that patients did not all have the same number of followup visits. Second, we only considered surgical failures and did not include patients with clinical or radiographic (impending) failure, so it is plausible that this study underestimates the failure rate of revision THA. Third, with longer followup the rate and the etiology of failure may alter. Finally, we lacked "standard" definitions for etiology of failure. It is possible that some of the aseptic failures were indeed due to infection and may have escaped detection using the available diagnostic modalities. Also, as the cause of failure for each case was extracted from the medical records and based on the judgment of the treating surgeon, it is possible that some inaccuracy or bias may have existed and the exact etiology for failure in some cases as recorded may not have been precisely accurate.

Our data suggest infection and instability were the two most common modes of failure after revision THA, comprising $30 \%$ and $25 \%$ of 256 failed revisions, respectively. In the study by Springer and coworkers [20], instability and aseptic loosening were the first two main modes of failure comprising $35 \%$ and $30 \%$ of the failures, respectively, followed by deep periprosthetic infection comprising $12 \%$ of the failures. Although the rate of indications for the index revision surgery in the Springer et al. study was comparable to ours, the rate of failures resulting from infection and loosening were different, which could be attributed to the difference in the time span of the studies. Although the average followup in that study is comparable to the current study ( 6 years versus 5.5 years), the time span of study by Springer et al. was relatively long at 20 years, whereas the cohort selected for our study are from a short period of 6 years [20]. So it is possible that due to changes in prosthesis design, such as bearing surface changes allowing the use of larger femoral heads and surgical techniques such as recognizing the importance of capsular closure, the etiology for failure in recent decades may differ considerably from earlier years. There are multiple other reasons that may clarify the discrepancy between our findings and the study by Springer et al. [20]. It is possible that different definitions for end point or modes of failure may have been selected. For example, wear leading to instability may have been denoted as "instability" in one study versus "wear or aseptic failure" in another. In fact, we considered reoperation in addition to revision as an end point for failure whereas Springer et al. considered only rerevision as the end point for failure which will, of course, lead to a higher rate of failure in our study [20]. Despite applying measures such as using larger femoral heads, inserting constrained or elevated rim liners, performing posterior capsular repair during a posterior approach that has decreased the incidence of dislocation after revision THA [1, 10, 18, 21], instability still constitutes a considerable number of failures after revision hip arthroplasty [20]. Dislocation occurs after $0.3 \%$ to $10 \%$ of primary THAs and after up to $28 \%$ of revision THAs [18]. Our study showed the rate of instability after revision surgery was $4.6 \%$ (63 hips out of 1366 revision THAs).

We found revisions for infection and instability had lower survival compared to the rest of our cohort. The overall survivorship of the cohort at 5 years was $83.3 \%$, but after separating the infection and instability groups the remaining cohort survival rate was $95.6 \%$. In some reports over $80 \%$ success rate has been noted for revision of infected THA by two-stage reimplantation. The low rate of survival of revision following infection in our study could be due to two reasons. First, we included the outcome of both single- and two-stage exchange arthroplasty and second, we considered any reoperation such as need for irrigation and débridement as failure also. Reoperations, such as closed reduction for dislocation, were considered as failure. The latter may explain why the success rate of revision for instability was lower at $75.9 \%$ in our study compared to a success rate of $86 \%$ in the study by Springer et al. [20].

We observed cause of failure "persisted" and the reason for failure of the original surgery was also the major reason for failure of cases that needed rerevision. For example, approximately one-fifth (22\%) of the revision THAs performed for infection failed subsequently because of infection. A $14 \%$ rate of failure for cases revised for instability was also the same for later failures. Although intuitive, this finding has not been described in previous studies as far as we are aware.

As etiology for failure of revision THA resulting from loosening and wear continues to decline because of advances in prosthetic design, availability of better bearing surfaces, and improvements in fixation techniques, infection is expected to be one of the key problems encountered in the future. We believe better strategies to prevent or effectively treat this dreaded complication are needed.

\section{References}

1. Alberton GM, High WA, Morrey BF. Dislocation after revision total hip arthroplasty: an analysis of risk factors and treatment options. J Bone Joint Surg Am. 2002;84:1788-1792.

2. Barrack RL. Economics of revision total hip arthroplasty. Clin Orthop Relat Res. 1995;319:209-214. 
3. Barrack RL, Hoffman GJ, Tejeiro WV, Carpenter LJ, Jr. Surgeon work input and risk in primary versus revision total joint arthroplasty. J Arthroplasty. 1995;10:281-286.

4. Barrack RL, Sawhney J, Hsu J, Cofield RH. Cost analysis of revision total hip arthroplasty. A 5-year followup study. Clin Orthop Relat Res. 1999;369:175-178.

5. Berbari EF, Hanssen AD, Duffy MC, Steckelberg JM, Ilstrup DM, Harmsen WS, Osmon DR. Risk factors for prosthetic joint infection: case-control study. Clin Infect Dis. 1998;27:1247-1254.

6. Bozic KJ, Kurtz SM, Lau E, Ong K, Vail TP, Berry DJ. The epidemiology of revision total hip arthroplasty in the United States. J Bone Joint Surg Am. 2009;91:128-133.

7. Crowe JF, Sculco TP, Kahn B. Revision total hip arthroplasty: hospital cost and reimbursement analysis. Clin Orthop Relat Res. 2003;413:175-182.

8. Della Valle CJ, Shuaipaj T, Berger RA, Rosenberg AG, Shott S, Jacobs JJ, Galante JO. Revision of the acetabular component without cement after total hip arthroplasty. A concise follow-up, at fifteen to nineteen years, of a previous report. J Bone Joint Surg Am. 2005;87:1795-1800.

9. Dorey F, Amstutz HC. Survivorship analysis in the evaluation of joint replacement. J Arthroplasty. 1986;1:63-69.

10. Hummel MT, Malkani AL, Yakkanti MR, Baker DL. Decreased dislocation after revision total hip arthroplasty using larger femoral head size and posterior capsular repair. J Arthroplasty. 2009;24(6 Suppl):73-76.

11. Kaplan EL, Meier P. Nonparametric estimation from incomplete data. J Am Stat Assoc. 1958;53:457-481.

12. Kurtz S, Ong K, Lau E, Mowat F, Halpern M. Projections of primary and revision hip and knee arthroplasty in the United States from 2005 to 2030. J Bone Joint Surg Am. 2007;89:780-785.
13. Lavernia CJ, Drakeford MK, Tsao AK, Gittelsohn A, Krackow KA, Hungerford DS. Revision and primary hip and knee arthroplasty. A cost analysis. Clin Orthop Relat Res. 1995;311: 136-141.

14. Levine DB, Cole BJ, Rodeo SA. Cost awareness and cost containment at the Hospital for Special Surgery. Strategies and total hip replacement cost centers. Clin Orthop Relat Res. 1995;311: 117-124.

15. Lie SA, Havelin LI, Furnes ON, Engesaeter LB, Vollset SE. Failure rates for 4762 revision total hip arthroplasties in the Norwegian Arthroplasty Register. J Bone Joint Surg Br. 2004;86: 504-509.

16. McCarthy JC, Lee JA. Complex revision total hip arthroplasty with modular stems at a mean of 14 years. Clin Orthop Relat Res. 2007;465:166-169.

17. Ong KL, Mowat FS, Chan N, Lau E, Halpern MT, Kurtz SM. Economic burden of revision hip and knee arthroplasty in Medicare enrollees. Clin Orthop Relat Res. 2006;446:22-28.

18. Parvizi J, Picinic E, Sharkey PF. Revision total hip arthroplasty for instability: surgical techniques and principles. J Bone Joint Surg Am. 2008;90:1134-1142.

19. Ritter MA, Carr KD, Keating EM, Faris PN, Bankoff DL, Ireland PM. Revision total joint arthroplasty: does medicare reimbursement justify time spent? Orthopedics. 1996;19:137139.

20. Springer BD, Fehring TK, Griffin WL, Odum SM, Masonis JL. Why revision total hip arthroplasty fails. Clin Orthop Relat Res. 2009;467:166-173.

21. Suh KT, Roh HL, Moon KP, Shin JK, Lee JS. Posterior approach with posterior soft tissue repair in revision total hip arthroplasty. $J$ Arthroplasty. 2008;23:1197-1203. 